\title{
A Survey on Expatriate Teachers in Southeast Asia's Intention to Leave due COVID-19
}

\author{
Authors \\ Anh-Duc Hoang, ${ }_{1, *}$, Ngoc-Thuy Ta ${ }_{1}$, Yen-Chi Nguyen $_{1}$,Cong-Kien Hoang, Tien-Trung \\ Nguyen $_{3}$, Hiep-Hung Pham 4 , Linh-Chi Nguyen 1 , Phuong-Thuc Doan 1 , Quynh-Anh Daor, \\ Viet-HungDinh $_{5}$
}

\section{Affiliations}

1. EdLab Asia Educational Research and Development Centre, Hanoi 100000, Vietnam

2. Hung Vuong University, Phu Tho 35000, Vietnam

3. Institute of Theoretical and Applied Research, Duy Tan University, Hanoi 10000, Vietnam

4. Phu Xuan University, Hue 4900o, Vietnam

5. University of Labour and Social Affairs, Hanoi 1000oo, Vietnam

\section{Corresponding author(s)}

Anh-Duc Hoang (duc@edlabasia.org)

\begin{abstract}
The COVID-19 pandemic exerted an adverse influence on the global education system, especially since starting school lockdown. The growth of teacher unemployment figures climbed double-digit and spawned these unexpected sequels. For instance, while native teachers seemed indisposed to leave the profession with the aim of seeking another more profited and seasonal jobs, many ex-pat teachers presented themselves with moving or stayed in dilemma in the way the government salvaged their situation. In preference with the ex-pat teacher's case, we elucidated further throughout an e-survey in the International Baccalaureate community on Facebook from 4 to 11 April 2020 for 18,000 ex-pat teachers, who are teaching in Southeast Asia. This dataset includes 307 responses of ex-pat teachers who are staying in Singapore, Thailand, Vietnam, the Philippines, and Indonesia during the pandemic. The dataset comprises (i) Survey partakers' Demographics; (ii) Ex-pat teachers' perceptions in the relation of national, regional and school plans were afoot to the pandemic; (iii) The degree of attachment of ex-pat teacher to their current society, the ex-pat community, friends, and families during the pandemic time; (iv) Ex-pat teachers' embryo intention to reconsider their current teaching location.
\end{abstract}

\section{Keywords}

Teacher Engagement; Teacher Retention; COVID-19; Southeast Asia; International School; Ex-pat Teacher; Education Management

\section{Specifications Table}




\begin{tabular}{|c|c|}
\hline Subject & Education, Education Management \\
\hline $\begin{array}{l}\text { Specific subject } \\
\text { area }\end{array}$ & Teacher retention, Teacher engagement \\
\hline Type of data & Raw data in excel file and analysed data \\
\hline $\begin{array}{l}\text { How data were } \\
\text { acquired }\end{array}$ & $\begin{array}{l}\text { Data was gathered using an online survey and converted into } \\
\text { the .xlsx format for formal analysis in SPSS v.20. }\end{array}$ \\
\hline Data format & $\begin{array}{l}\text { Raw } \\
\text { Analyzed }\end{array}$ \\
\hline $\begin{array}{l}\text { Parameters for } \\
\text { data collection }\end{array}$ & $\begin{array}{l}\text { This research focuses on ex-pat teachers who are teaching in } \\
\text { several Southeast Asia countries: Singapore, Thailand, } \\
\text { Vietnam, the Philippines, and Indonesia. }\end{array}$ \\
\hline $\begin{array}{l}\text { Description of data } \\
\text { collection }\end{array}$ & $\begin{array}{l}\text { An online survey has been distributed throughout the } \\
\text { International Baccalaureate community on Facebook (18,ooo } \\
\text { ex-pat teachers worldwide) and mainly ranged within ex-pat } \\
\text { teachers who are working in Southeast Asia. }\end{array}$ \\
\hline $\begin{array}{l}\text { Data source } \\
\text { location }\end{array}$ & 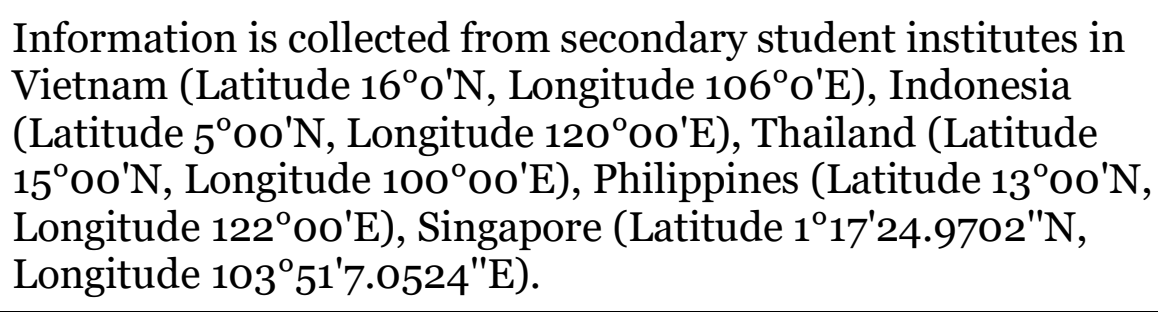 \\
\hline Data accessibility & $\begin{array}{l}\text { Repository name: Harvard Dataverse } \\
\text { Data identification number: } \\
\text { Direct URL to data: https://doi.org/10.7910/DVN/ZB2DNH, } \\
\text { Harvard Dataverse, V1 }\end{array}$ \\
\hline
\end{tabular}

\section{Value of the Data}

- The dataset heralds further research into these underlying reasons why ex-pat teachers no longer keep their teaching location stayed.

- Policymakers, schools, or even business managers can utilize this dataset to address brain drain-related phenomenon.

- This dataset can be accessed to more corrective courses of action, which bring teachers into perceiving the policy decision.

- The dataset offers an additional contribution to publication reviews regarding the policy's influence extended towards teacher involvement. 
- The dataset produces a scale model exploring ex-pat teachers' changing perceptions about their current working regions, especially when these national politics formulate different policies during the COVID-19 pandemic.

\section{Data Description}

Teacher retention and teacher engagement are strong influencers in educational institutions, especially in terms of students' academic achievement [1,2]. Due to the COVID-19 pandemic, schools around the world had to choose distance learning with many changes in ways of teaching and learning, thus native and ex-pat teachers were both affected $[3,4]$. In addition, this unexpected digital transformation creates many educational problems related to learning and teaching demand [5]. This dataset focuses on ex-pat teachers' engagement and intention to leave, which is an expansion of our recent research about Vietnamese teachers' perceptions and student's learning habits during the pandemic $[6,7,8]$.

This dataset contains two main parts, the first part is demographic information, and the second part reports on teachers' perspective and intention. The former includes teachers' gender, nationality, teaching country, teaching subject and grade, school type, teaching qualification and experience, and participants' income. The later part concerns three main issues related to the pandemic: (i) Policy and regulation toward ex-pat teachers; (ii) Ex-pat teachers' engagement with various communities; and (iii) Intention to leave of expat teachers. The above variables can be used to study teacher retention, teacher engagement, impacts of policy, and teachers' salary. Finally, the full survey, code, and measurement parameters for all variables can be found on Harvard Dataverse [9].

Table 1. Descriptive Statistics of Participant's Demographics

\begin{tabular}{|c|c|c|c|c|c|c|c|}
\hline & \multirow{2}{*}{ Intend } & \multirow[t]{2}{*}{$\mathbf{N}$} & \multirow[t]{2}{*}{ Mean } & \multirow{2}{*}{$\begin{array}{l}\text { Std. } \\
\text { Deviation }\end{array}$} & \multirow{2}{*}{$\begin{array}{l}\text { Std. } \\
\text { Error }\end{array}$} & \multicolumn{2}{|c|}{$\begin{array}{l}\text { 95\% Confidence } \\
\text { Interval for } \\
\text { Mean }\end{array}$} \\
\hline & & & & & & $\begin{array}{l}\text { Lower } \\
\text { Bound }\end{array}$ & $\begin{array}{l}\text { Upper } \\
\text { Bound }\end{array}$ \\
\hline \multirow{2}{*}{ Gender } & Male & 131 & 2.735 & 0.890 & 0.078 & 2.581 & 2.889 \\
\hline & Female & 176 & 2.801 & 0.880 & 0.066 & 2.670 & 2.932 \\
\hline \multirow{5}{*}{$\begin{array}{l}\text { Current } \\
\text { country of } \\
\text { teaching }\end{array}$} & Indonesia & 34 & 3.441 & 0.700 & 0.120 & 3.197 & 3.685 \\
\hline & Thailand & 66 & 2.904 & 0.882 & 0.109 & 2.687 & 3.121 \\
\hline & Philippines & 51 & 3.157 & 0.823 & 0.115 & 2.925 & 3.388 \\
\hline & Vietnam & 121 & 2.413 & 0.859 & 0.078 & 2.259 & 2.568 \\
\hline & Singapore & 35 & 2.562 & 0.503 & 0.085 & 2.389 & 2.735 \\
\hline \multirow{3}{*}{ School type } & Public school & 50 & 2.747 & 0.856 & 0.121 & 2.503 & 2.990 \\
\hline & Private school & 227 & 2.819 & 0.860 & 0.057 & 2.707 & 2.932 \\
\hline & $\begin{array}{l}\text { Extracurricular Edu } \\
\text { Center }\end{array}$ & 29 & 2.448 & 1.070 & 0.199 & 2.041 & 2.855 \\
\hline \multirow{2}{*}{ Nationality } & $\begin{array}{l}\text { Australia and New } \\
\text { Zealand }\end{array}$ & 63 & 2.963 & 0.741 & 0.093 & 2.776 & 3.150 \\
\hline & Europe & 106 & 2.840 & 0.932 & 0.091 & 2.660 & 3.019 \\
\hline
\end{tabular}




\begin{tabular}{|c|c|c|c|c|c|c|c|}
\hline & South Africa & 11 & 2.091 & 0.870 & 0.262 & 1.506 & 2.676 \\
\hline & US and Canada & 107 & 2.732 & 0.888 & 0.086 & 2.562 & 2.902 \\
\hline & Others & 20 & 2.417 & 0.786 & 0.176 & 2.049 & 2.785 \\
\hline \multirow{4}{*}{ Grade level } & Kindergarten & 14 & 2.286 & 0.714 & 0.191 & 1.873 & 2.698 \\
\hline & $\begin{array}{l}\text { Lower secondary } \\
\text { school }\end{array}$ & 111 & 2.775 & 0.841 & 0.080 & 2.617 & 2.933 \\
\hline & $\begin{array}{l}\text { Upper secondary } \\
\text { school }\end{array}$ & 74 & 2.685 & 0.846 & 0.098 & 2.489 & 2.881 \\
\hline & Primary school & 108 & 2.895 & 0.950 & 0.091 & 2.714 & 3.076 \\
\hline \multirow{3}{*}{ Degree } & BA in Education & 180 & 2.928 & 0.787 & 0.059 & 2.812 & 3.044 \\
\hline & MA in Education & 78 & 2.628 & 0.912 & 0.103 & 2.423 & 2.834 \\
\hline & Teaching certificate & 49 & 2.435 & 1.044 & 0.149 & 2.136 & 2.735 \\
\hline \multirow{5}{*}{$\begin{array}{l}\text { Experience } \\
\text { at the } \\
\text { current } \\
\text { country }\end{array}$} & Less than a year & 33 & 2.485 & 1.014 & 0.177 & 2.125 & 2.844 \\
\hline & 1 year & 92 & 3.069 & 0.729 & 0.076 & 2.918 & 3.220 \\
\hline & 2 years & 106 & 3.000 & 0.735 & 0.071 & 2.859 & 3.141 \\
\hline & 3 years & 29 & 2.483 & 0.699 & 0.130 & 2.217 & 2.749 \\
\hline & More than 3 years & 47 & 2.064 & 0.987 & 0.144 & 1.774 & 2.354 \\
\hline \multirow{7}{*}{$\begin{array}{l}\text { Income } \\
\text { before } \\
\text { covid-19 }\end{array}$} & Less than 1500 USD & 15 & 2.311 & 0.636 & 0.164 & 1.959 & 2.663 \\
\hline & 1500 1999 USD & 28 & 2.512 & 1.389 & 0.263 & 1.973 & 3.051 \\
\hline & 2000 2499 USD & 46 & 2.696 & 0.913 & 0.135 & 2.425 & 2.967 \\
\hline & 2500 2999 USD & 110 & 3.245 & 0.663 & 0.063 & 3.120 & $3 \cdot 371$ \\
\hline & 3000 3499 USD & 81 & 2.646 & 0.661 & 0.073 & 2.500 & 2.792 \\
\hline & 3500 3999 USD & 16 & 1.917 & 0.627 & 0.157 & 1.583 & 2.251 \\
\hline & More than 4000 USD & 11 & 1.848 & 0.480 & 0.145 & 1.526 & 2.171 \\
\hline \multirow{7}{*}{$\begin{array}{l}\text { Income } \\
\text { during } \\
\text { covid-19 }\end{array}$} & Less than 1500 USD & 63 & 2.418 & 1.007 & 0.127 & 2.164 & 2.672 \\
\hline & 1500 1999 USD & 36 & 3.102 & 0.956 & 0.159 & 2.779 & 3.425 \\
\hline & 2000 2499 USD & 98 & 3.163 & 0.750 & 0.076 & 3.013 & $3 \cdot 314$ \\
\hline & 2500 2999 USD & 64 & 2.750 & 0.669 & 0.084 & 2.583 & 2.917 \\
\hline & 3000 3499 USD & 24 & 2.542 & 0.537 & 0.110 & 2.315 & 2.768 \\
\hline & 3500 3999 USD & 12 & 1.806 & 0.658 & 0.190 & 1.387 & 2.224 \\
\hline & More than 400o USD & 10 & 1.867 & 0.502 & 0.159 & 1.508 & 2.226 \\
\hline \multirow{7}{*}{$\begin{array}{l}\text { Income } \\
\text { after covid- } \\
19\end{array}$} & Less than 1500 USD & 23 & 2.406 & 0.899 & 0.187 & 2.017 & 2.794 \\
\hline & 1500 1999 USD & 28 & 2.607 & 1.264 & 0.239 & 2.117 & 3.097 \\
\hline & 2000 2499 USD & 30 & 2.433 & 0.889 & 0.162 & 2.101 & 2.765 \\
\hline & 2500 2999 USD & 106 & 3.302 & 0.638 & 0.062 & 3.179 & 3.425 \\
\hline & 3000 3499 USD & 87 & 2.655 & 0.658 & 0.071 & 2.515 & 2.795 \\
\hline & 3500 3999 USD & 19 & 1.982 & 0.662 & 0.152 & 1.663 & 2.301 \\
\hline & More than 4000 USD & 14 & 2.238 & 0.999 & 0.267 & 1.661 & 2.815 \\
\hline & Total & 307 & 2.773 & 0.883 & 0.050 & 2.674 & 2.872 \\
\hline
\end{tabular}

Table 2. Correlations between variables and ex-pat teacher's intention to leave the current country 


\begin{tabular}{|l|l|l|l|l|l|}
\hline & $\begin{array}{l}\text { Sum of } \\
\text { Squares }\end{array}$ & df & $\begin{array}{l}\text { Mean } \\
\text { Square }\end{array}$ & F & \\
\hline Gender & .325 & 1 & .325 & .416 & .520 \\
\hline $\begin{array}{l}\text { Current country of } \\
\text { teaching }\end{array}$ & 41.050 & 4 & 10.262 & 15.677 & $.000^{* * * *}$ \\
\hline School type & 3.581 & 2 & 1.790 & 2.307 & .101 \\
\hline Nationality & 10.580 & 4 & 2.645 & 3.501 & $.008^{* *}$ \\
\hline Grade level & 5.511 & 3 & 1.837 & 2.386 & $.069^{*}$ \\
\hline Degree & 11.533 & 2 & 5.766 & 7.715 & $.002^{* * *}$ \\
\hline $\begin{array}{l}\text { Experience at the current } \\
\text { country }\end{array}$ & 42.334 & 4 & 10.584 & 16.273 & $.000^{* * *}$ \\
\hline Income before covid-19 & 52.377 & 6 & 8.730 & 14.052 & $.000^{* * *}$ \\
\hline Income during covid-19 & 47.523 & 6 & 7.921 & 12.426 & $.000^{* * *}$ \\
\hline Income after covid-19 & 54.071 & 6 & 9.012 & 14.639 & $.000^{* * *}$ \\
\hline
\end{tabular}

* Correlation is significant at the 0.05 level; ${ }^{* *}$ Correlation is significant at the 0.01 level; ${ }^{* * *}$ Correlation is significant at the o.0o1 level

Table 3. Significant of ANOVA analyses

\begin{tabular}{|l|c|c|c|}
\hline \multicolumn{1}{|c|}{ Variable } & $\begin{array}{c}\text { Sig of } \\
\text { Homogeneity } \\
\text { test }\end{array}$ & $\begin{array}{c}\text { Sig of } \\
\text { ANOVA test }\end{array}$ & $\begin{array}{c}\text { Sig of Robust } \\
\text { Tests of Equality } \\
\text { of Means }\end{array}$ \\
\hline Nationality & .377 & .008 & \\
\hline $\begin{array}{l}\text { Current country of } \\
\text { teaching }\end{array}$ & .024 & & .000 \\
\hline Teaching qualification & .038 & & .002 \\
\hline $\begin{array}{l}\text { Experience at current } \\
\text { country }\end{array}$ & .120 & .000 & \\
\hline Income before COVID-19 & .000 & & .000 \\
\hline Income during COVID-19 & .003 & & .000 \\
\hline Income after COVID-19 & .000 & & .000 \\
\hline
\end{tabular}

Table 4. Differences in Teachers' Intention of Leaving during the COVID-19 Pandemic among Different Demographics (ANOVA analysis)

\begin{tabular}{|l|l|l|l|l|l|l|}
\hline & & $\begin{array}{l}\text { Sum of } \\
\text { Squares }\end{array}$ & df & $\begin{array}{l}\text { Mean } \\
\text { Square }\end{array}$ & F & Sig. \\
\hline Nationality & $\begin{array}{l}\text { Between } \\
\text { Groups }\end{array}$ & 10.580 & 4 & 2.645 & 3.501 & .008 \\
\cline { 2 - 7 } & $\begin{array}{l}\text { Within } \\
\text { Groups }\end{array}$ & 228.166 & 302 & .756 & & \\
\cline { 2 - 7 } & Total & 238.746 & 306 & & & \\
\hline
\end{tabular}




\begin{tabular}{|c|c|c|c|c|c|c|}
\hline \multirow{3}{*}{$\begin{array}{l}\text { Current } \\
\text { country of } \\
\text { teaching }\end{array}$} & $\begin{array}{l}\text { Between } \\
\text { Groups }\end{array}$ & 41.050 & 4 & 10.262 & 15.677 & .000 \\
\hline & $\begin{array}{l}\text { Within } \\
\text { Groups }\end{array}$ & 197.697 & 302 & .655 & & \\
\hline & Total & 238.746 & 306 & & & \\
\hline \multirow[t]{3}{*}{ Degree } & $\begin{array}{l}\text { Between } \\
\text { Groups }\end{array}$ & 11.533 & 2 & 5.766 & 7.715 & .001 \\
\hline & $\begin{array}{l}\text { Within } \\
\text { Groups }\end{array}$ & 227.213 & 304 & .747 & & \\
\hline & Total & 238.746 & 306 & & & \\
\hline \multirow{3}{*}{$\begin{array}{l}\text { Experience } \\
\text { at the } \\
\text { current } \\
\text { country }\end{array}$} & $\begin{array}{l}\text { Between } \\
\text { Groups }\end{array}$ & 42.334 & 4 & 10.584 & $\begin{array}{l}16.27 \\
3\end{array}$ & .000 \\
\hline & $\begin{array}{l}\text { Within } \\
\text { Groups }\end{array}$ & 196.412 & 302 & .650 & & \\
\hline & Total & 238.746 & 306 & & & \\
\hline \multirow{3}{*}{$\begin{array}{l}\text { Income } \\
\text { before } \\
\text { covid-19 }\end{array}$} & $\begin{array}{l}\text { Between } \\
\text { Groups }\end{array}$ & 52.377 & 6 & 8.730 & $\begin{array}{l}14.05 \\
2\end{array}$ & .000 \\
\hline & $\begin{array}{l}\text { Within } \\
\text { Groups }\end{array}$ & 186.369 & 300 & .621 & & \\
\hline & Total & 238.746 & 306 & & & \\
\hline \multirow{3}{*}{$\begin{array}{l}\text { Income } \\
\text { during } \\
\text { covid-19 }\end{array}$} & $\begin{array}{l}\text { Between } \\
\text { Groups }\end{array}$ & $47 \cdot 523$ & 6 & 7.921 & $\begin{array}{l}12.42 \\
6\end{array}$ & .000 \\
\hline & $\begin{array}{l}\text { Within } \\
\text { Groups }\end{array}$ & 191.223 & 300 & .637 & & \\
\hline & Total & 238.746 & 306 & & & \\
\hline \multirow{3}{*}{$\begin{array}{l}\text { Income } \\
\text { after covid- } \\
19\end{array}$} & $\begin{array}{l}\text { Between } \\
\text { Groups }\end{array}$ & 54.071 & 6 & 9.012 & $\begin{array}{l}14.63 \\
9\end{array}$ & .000 \\
\hline & $\begin{array}{l}\text { Within } \\
\text { Groups }\end{array}$ & 184.675 & 300 & .616 & & \\
\hline & Total & 238.746 & 306 & & & \\
\hline
\end{tabular}

Table 5. Robust Tests of Equality of Means toward Teacher's Intention of Leaving

\begin{tabular}{|l|l|l|l|l|l|}
\hline & Welch & Statistic & df1 & df2 & Sig. \\
\hline $\begin{array}{l}\text { Current country of } \\
\text { teaching }\end{array}$ & & 17.438 & 4 & 116.594 & .000 \\
\hline Degree & & 6.681 & 2 & 105.788 & .002 \\
\hline $\begin{array}{l}\text { Income before } \\
\text { covid-19 }\end{array}$ & 21.872 & 6 & 59.158 & .000 \\
\hline $\begin{array}{l}\text { Income during } \\
\text { covid-19 }\end{array}$ & & 15.884 & 6 & 61.172 & .000 \\
\hline $\begin{array}{l}\text { Income after covid- } \\
\mathbf{1 9}\end{array}$ & & 17.776 & 6 & 67.821 & .000 \\
\hline
\end{tabular}




\section{Experimental Design, Materials, and Methods}

Firstly, four experts in K-12 international education and organizational behavior were asked to pretest the validity of the assessments. Then we implemented a pilot study including 50 observations, before distributing the survey online within a Facebook community named International Baccalaureate from 4th to 11th April 2020. We only collect data from ex-pats who were teaching in Southeast Asia and recorded 528 accesses on the survey link. Among those, teachers from Indonesia, Philippines, Singapore, Thailand, and Vietnam accounted for the majority; thus, 36 responses were deleted since they were from other countries. Finally, after cleaning the dataset, there were 307 observations valid for further analysis.

Table 1 is the descriptive statistics of participants' demographics. Table 2 shows the relationship between ex-pat teachers' intention to leave and various indicators. The differences between participants' retention among demographic variables are examined and presented through ANOVA analysis. Specifically, Table 3 is the summary of ANOVA analysis's significance, Table 4 is the more detailed results of between and within groups, and Table 5 shows specific robust test' results.

This dataset uses mainly five-point Likert scale to examine the impacts of various factors on ex-pat teachers' retention. Based on this dataset, some research can be carried out to study the relationships between teacher engagement and external policy on teachers' intention to leave (INTEND). First, teacher engagement (ENGAGE) is considered to have long-term influence over schools and societies [10]. This relationship becomes even more substantial and more complicated, especially in this era of globalization, when ex-pat teachers frequently face multiculturalism [11]. In this dataset, teacher engagement can be indicated by activities and communication among inter-related stakeholders [12], such as local communities (ENGAGE_LOCAL), ex-pat communities (ENGAGE_EXPAT) and families and friends at home (ENGAGE_HOME). Consequently, the relationship between teacher engagement and teacher retention can be found by using the regression model (1). Similarly, the impact of policy on teacher retention should also be investigated [13, 14], 1985) as in model (2). In the questionnaire, the policy and regulation (POLICY) under examination are national policy (POLI_NATION), regional policy (POLI_REGION), and school policy (POLI_SCHOOL). Finally, model (3) can also lead to significant results. However, as policies can affect teacher engagement [15], researchers may consider using instrumental variables.

$$
\begin{gathered}
\text { INTEND } \sim \beta \mathrm{o}+\beta 1^{*} \text { ENGAGE }+\mathrm{u}(1) \\
\text { INTEND } \sim \beta \mathrm{O}+\beta 1^{*} \text { POLICY }+\mathrm{u}(2) \\
\text { INTEND } \sim \beta \mathrm{O}+\beta 1^{*} \text { ENGAGE }+\beta 2^{*} \text { POLICY }+\mathrm{u}(3)
\end{gathered}
$$

\section{Acknowledgments}


A great thanks to all teachers who participated in this study, school leaders and instructional coach, who contributed to elevating the data collection process.

\section{Competing Interests}

The authors declare that they have no known competing financial interests or personal relationships which have, or could be perceived to have, influenced the work reported in this article.

\section{References}

[1] McLaurin, S. E., Smith, W., \& Smillie, A. (2009). Teacher Retention: Problems and Solutions. Online submission.

[2] Guthrie, J. T., Dreher, M. J., \& Baker, L. I. N. D. A. (2000). Why teacher engagement is important to student achievement. Engaging young readers: Promoting achievement and motivation, 309-320.

[3] Nguyen, L. (2020). Nghỉ học để phòng dịch Covid-19: Học trực tuyến có đóng phí? [School closure due to COVID-19: Do parents have to pay tuition for online learning?]. Youth Newspaper. https://thanhnien.vn/giao-duc/nghi-hoc-de-phong-dich-covid-19hoc-truc-tuyen-co-dong-phi-1197352.html

[4] Nguyen, T., (2020). 150 cơ sở giáo dục tư thục 'cầu cứu' vì đóng cửa do dịch Covid 19. [150 private educational institutions ask for help because of the closure due to Covid 19]. Youth Newspaper. https://thanhnien.vn/giao-duc/150-co-so-giao-duc-tu-thuc-caucuu-vi-dong-cua-do-dich-covid-19-1191469.html

[5] Hodges, C., Moore, S., Lockee, B., Trust, T., \& Bond, A. (2020). The difference between emergency remote teaching and online learning. EDUCAUSE Review. https://er. educause. $\quad$ edu/articles/2020/3/the-difference-between-emergency-remoteteachingand-online-learning .

[6] Tran, T., Hoang, A. D., Nguyen, T. T., Dinh, V.H., Nguyen, Y. C., Pham, H. H. (2020). Dataset of Vietnamese student's learning habits during COVID-19. Data in Brief, vol. 30. $105682 \mathrm{https}: / /$ doi.org/10.1016/j.dib.2020.105682

[7] Tran, T., Hoang, A. D., Nguyen, Y. C., Nguyen, L. C., Ta, N. T., Pham, Q. H., Pham, C. X., Le, Q. A., Dinh, V. H., Nguyen, T. T. (2020). Toward Sustainable Learning during School Suspension: Socioeconomic, Occupational Aspirations, and Learning Behavior of Vietnamese Students during COVID-19. Sustainability, 12(10):4195. https://doi.org/10.3390/su12104195

[8] Vu, C. T., Hoang, A. D., Than, V. Q., Nguyen, M. T., Dinh, V. H., Le, Q. A. T., ... \& Nguyen, Y. C. (2020). Dataset of Vietnamese teachers' perspectives and perceived support during the COVID-19 pandemic. Data in Brief, 105788.

[9] [dataset] Hoang, Anh-Duc, 2020, "Survey on ex-pat teachers' intention to leave due to COVID-19", https://doi.org/10.7910/DVN/ZB2DNH, Harvard Dataverse, V1.

[10] Louis, K. S., \& Smith, B. (1991). Restructuring, teacher engagement and school culture: Perspectives on school reform and the improvement of teacher's work. School Effectiveness and School Improvement, 2(1), 34-52.

[11] Pekerti, A. A., Vuong, Q. H., \& Napier, N. K. (2017). Double edge experiences of expatriate acculturation. Journal of Global Mobility.

[12] Cropanzano, R., \& Mitchell, M. S. (2005). Social exchange theory: An

interdisciplinary review. Journal of management, 31(6), 874-900. 
[13] Ajzen, I. (1985). From intentions to actions: A theory of planned behavior. In Action control (pp. 11-39). Springer, Berlin, Heidelberg.

[14] Cochran-Smith, M. (2004). Stayers, leavers, lovers, and dreamers: Insights about teacher retention.

[15] Tripney, J., Gough, D., Sharples, J., Lester, S., \& Bristow, D. (2018). Promoting teacher engagement with research evidence. 